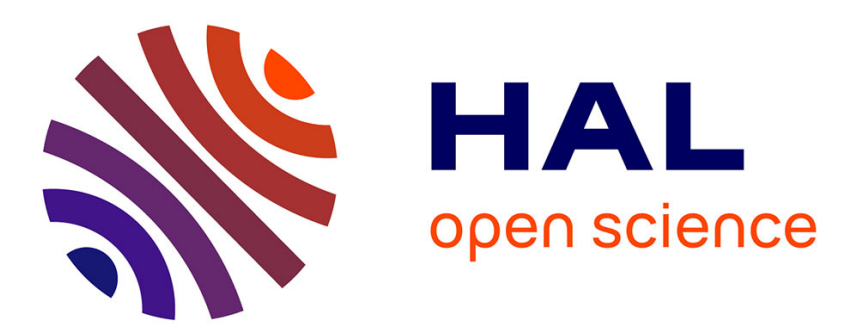

\title{
Le souci du territoire, les groupes locaux de traitement de la délinquance
}

Anne Wyvekens

\section{To cite this version:}

Anne Wyvekens. Le souci du territoire, les groupes locaux de traitement de la délinquance. Les Annales de la Recherche Urbaine, 1999, Au risque des espaces publics, 83 (1), pp.81-88. 10.3406/aru.1999.2252 . halshs-02270048

\section{HAL Id: halshs-02270048 \\ https://shs.hal.science/halshs-02270048}

Submitted on 23 Aug 2019

HAL is a multi-disciplinary open access archive for the deposit and dissemination of scientific research documents, whether they are published or not. The documents may come from teaching and research institutions in France or abroad, or from public or private research centers.
L'archive ouverte pluridisciplinaire HAL, est destinée au dépôt et à la diffusion de documents scientifiques de niveau recherche, publiés ou non, émanant des établissements d'enseignement et de recherche français ou étrangers, des laboratoires publics ou privés. 


\section{Le souci du territoire, les groupes locaux de traitement de la} délinquance

Anne Wyvekens

\section{Citer ce document / Cite this document :}

Wyvekens Anne. Le souci du territoire, les groupes locaux de traitement de la délinquance. In: Les Annales de la recherche urbaine, $\mathrm{N}^{\circ} 83-84,1999$. Au risque des espaces publics. pp. 81-88;

doi : https://doi.org/10.3406/aru.1999.2252

https://www.persee.fr/doc/aru_0180-930x_1999_num_83_1_2252

Fichier pdf généré le 23/04/2018 


\title{
Résumé
}

Le pouvoir judiciaire se pose en partenaire actif du traitement de la peur dans les grands ensembles, notamment dans les groupes locaux de traitement de la délinquance, inventés en Seine Saint Denis. Leurs interventions médiatrices entre les délinquants, leurs familles et les institutions s'efforcent de réduire l'asservissement de l'espace public à la loi du plus fort. Recréer des repères, marquer des limites, démêler l'écheveau des provocations, toutes ces actions de longue durée cherchent à restaurer le respect de l'autre et à développer le dialogue entre gens différents.

\begin{abstract}
Territorial concerns. Local groups come to grips with delinquency

The judiciary sets itself up as an active partner to address fear in large housing estates, mainly in local delinquency control groups invented in Seine Saint Denis near Paris. These groups act as mediators between delinquents, their families and the institutions and aim to reduce the subservience of the public space to the law of the strongest. Long-term actions enable people to find their bearings, mark out limits, contend with provocation, seek to restore respect for others and develop dialogue with people who are different.
\end{abstract}

\section{Resumen}

La preocupación del territorio. Los grupos locales de tratamiento de la delincuencia

El poder judicial se presenta como asociado activo del tratamiento del miedo en los grandes conjuntos de vivienda, en particular en los grupos locales de tratamiento de la delincuencia, inventados en Seine-Saint Denis, a las afueras de París. Sus intervenciones de mediación entre los delincuentes, sus familias y las instituciones se esfuerzan por reducir el sujeción del espacio público a la ley del más fuerte. Recrear hitos, marcar límites, terminar con la cadena sinfín de las provocaciones, son todas acciones a largo plazo que intentan restaurar el respeto del otro y desarrollar el diálogo entre gentes diferentes.

\section{Zusammenfassung}

Das Territorium schützen

Kommunale Gruppen gegen die Straffalligkeit

Die Justiz tritt als aktiver Partner bei dem Kampf gegen die Angst in den großen Wohnblocks auf — vor allem im Rahmen der im französischen Departement Seine-Saint Denis erfundenen «Kommunalgruppen gegen Straffälligkeit ». Bei ihren Vermittlungsversuchen zwischen den Delinquenten, ihren Familien und den Institutionen geht es darum, die Unterwerfung des Öffentlichen Raums unter das Gesetz des Stärkeren zu reduzieren. Orientierungspunkte geben, Grenzen setzen, verwickelte Provokationen entwirren - all diese nur langfristig wirksamen Unternehmungen versuchen, den Respekt vor dem Anderen wiederherzustellen und den Dialog unter Ungleichen zu entwickeln. 


\section{LE SOUCI DU TERRITOIRE}

\section{LES GROUPES LOCAUX DE TRAITEMENT DE LA DELINQUANCE}

D epuis le rapport Bonnemaison, de Conseils communaux de prévention de la délinquance (CCPD) en maisons de justice, de "justice de proximité " en "politique judiciaire de la ville ", la justice a peu à peu fait sien le double enjeu, local et partenarial, des politiques de la ville. Si elle n'est entrée qu'avec circonspection dans la dynamique collective initiée par les CCPD, si son premier investissement de proximité est demeuré dans un premier temps très " immobilier " avec les maisons de justice, les deux idées ont progressivement fait leur chemin, au travers de divers dispositifs. Les "groupes locaux de traitement de la délinquance" (GLTD), expérimentés puis développés dans le ressort du tribunal de Bobigny, innovent par exemple aussi bien sur le plan de l'agencement du partenariat que sur celui de la façon d'intervenir sur un site. Les rapports entre les partenaires évoluent de façon telle, dans ces groupes, que la production de ceux-ci s'en trouve infléchie : avec les GLTD, au lieu de " traiter la délinquance ", l'institution judiciaire s'engage, avec ses partenaires non pénaux, dans une démarche consistant à s'intćresser à l'espace. Pour l'expliciter, nous avons mobilisé, avec Jacques Donzelot, les notions de " magistrature sociale " et de " souci du territoire ».

\section{Qu'est-ce qu'un groupe local de traitement de la délinquance?}

Le GLTD est un dispositif judiciaire : c'est le parquet qui le crée et qui le dirige. C'est un dispositif d'exception : il concerne des sites particulièrement dégradés, "hors-droit " ou en voie de le devenir, avec pour objectif de " rendre ceux-ci à des conditions de vie supportables». Dans cette perspective, il se veut temporaire : il est voué à être après six mois ou un an, "mis en sommeil ", de façon que le parquet puisse alors réinvestir, de la même manière, un autre site.

Le GLTD fonctionne sur un principe de double détente. Il consiste en premier lieu dans le déploiement, sur les sites en question, d'une action publique (policière et judiciaire) renforcée. Le parquet de Bobigny s'attache à rendre visible et à systématiser la réponse pénale. La visibilisation, ce sont en particulier les fameux contrôles d'identité « sur réquisition du parquet ", menés régulièrement dans les immeubles ou les centres commerciaux, destinés à montrer, précisément, que le quartier n'est pas " hors-droit », que le territoire n'appartient pas à ceux qui tentent d'y faire régner leur ordre à eux. C'est également un renforcement de l'îlotage policier, des passages de la Brigade anti-criminalité.

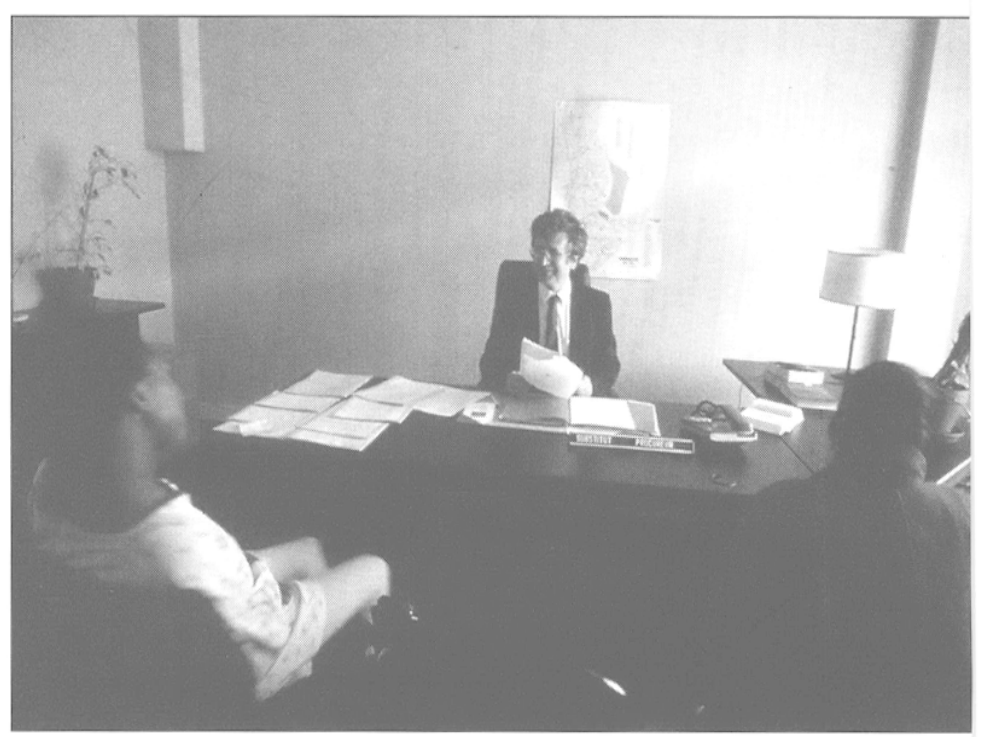

Maison de la Justice et du Droit, Sarcelles.

Quant à la systématisation, le parquet reprend un principe en vigueur pour les mineurs dans l'ensemble du ressort : aucun incident, qu'il soit petit ou grand, ne reste sans réaction. Pour y arriver, les magistrats s'emploient à récolter un maximum d'informations concernant le site. C'est là, dans un objectif d'abord pénal donc, que s'initie le partenariat. Chaque acteur local est interpellé par le parquet, qui lui demande de contribuer, de là où il se trouve, à une meilleure connaissancc, pour un traitement plus soutenu, des faits survenus sur le site. Cela commence bien sûr par la police, invitée à affiner ses informations, à communiquer des listes quotidiennes d'incidents. Cela continue avec l'Éducation nationale, ici aussi dans le prolongement d'une politique bien installée en Seine-Saint-Denis, qui vise à systématiser le signalement, à la police et au parquet, des incidents survenus en milieu scolaire. Les bailleurs sont également mis à contribution, les travailleurs sociaux, les municipalités, puis éventuellement les centres commerciaux, les sociétés de transport.

Les Annales de la Recherche Urbaine n" 83-84, 0180-930-LX-99/83-84/p. 81-88 @ METL. 
Le second volet consiste, toujours sous la houlette du parquet, à ce que l'ensemble des acteurs non pénaux prenne, chacun dans son domaine de compétence, le relais de cette action publique renforcée. Lidée qui sous-tend cet aspect de la démarche est que la répression ne peut rien à elle seule, si ce n'est engendrer le déplacement de la délinquance en un lieu moins

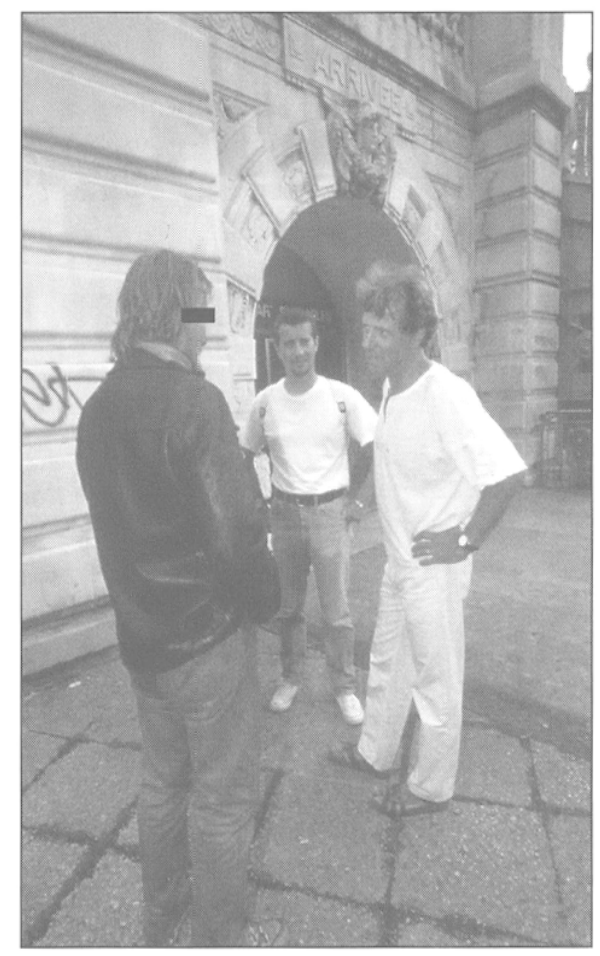

Éducateurs et SDF, le partage de la rue.

exposé aux foudres pénales. Mais ce qu'elle peut, c'est constituer le point de départ, et le point d'appui, d'une réappropriation du site par les autres acteurs. Le parquet, avec la police, vient montrer de façon forte que le quartier n'est pas hors-droit. C'est ensuite au tour des autres partenaires, appuyés sur le parquet, de reprendre leur place, de réinvestir chacun sa compétence propre pour assurer sur le site les régulations intermédiaires. Le GLTD est alors le lieu, ou le support, de mise en œuvre d'actions diverses mobilisant, ensemble ou séparément, les différents acteurs : grand nettoyage du site, création d'une maison des parents, installation de correspondants de nuit, interventions en milieu scolaire pour la prévention du racket, etc.

\section{Partenariat et territoire}

L'expression de " magistrature sociale " a été empruntée à Isabelle Astier ${ }^{1}$, qui l'utilise dans son analyse des commissions locales d'insertion pour décrire un mode de prise de décision dans lequel la participation de divers types d'acteurs permet d'ajuster attribution d'un droit et prise en compte de situations concrètes. Notre analyse lui donne un sens différent. Inspirée d'abord par l'origine judiciaire d'un dispositif où le magistrat (du parquet) occupe une position centrale, elle joue sur le double sens du terme de magistrature : son sens restrictif, judiciaire, et son sens extensif, désignant l'ensemble de ceux qui détiennent l'autorité. Car si le groupe est porté par le magistrat, il est également le lieu d'exercice d'un pouvoir de décision plus large, reconnu et confié à un ensemble d'acteurs. La magistrature de ceux-ci, leur autorité donc, est rendue possible par le soutien que lui apporte le magistrat. "Magistrature sociale " désigne alors une forme de prise en charge des questions de sécurité dont la première caractéristique est le partage, la répartition de compétences qu'elle suppose. Cette magistrature sociale se décline sous deux aspects. Le déclenchement de la démarche prend la forme d'un ensemble d'échanges bilatéraux, entre le parquet et chacun des acteurs locaux. A partir de ces échanges, on voit se développer peu à peu, dans la durée, une scène collective où l'ensemble des acteurs se retrouve et où les positions des uns rencontrent et font évoluer celles des autres.

Le groupe se constitue à partir de contacts entre le parquet et chacun des partenaires. Le magistrat interpelle ceux-ci au départ pour obtenir de l'information, voire une contribution plus ou moins directe à son œuvre de répression. Cette interpellation initiale ne va pas de soi : les différents acteurs non pénaux, chacun pour des raisons qui lui sont propres, répugnent à entrer dans une logique pénale. Les enseignants et les travailleurs sociaux ne veulent pas passer auprès des jeunes pour des "donneurs", les bailleurs sociaux considèrent que la sécurité est l'affaire de l'État, les municipalités sont toujours ambivalentes par rapport à une implication dans le sécuritaire. Il faut donc que le parquet réalise un travail de conviction, adapté à chaque acteur. A partir de là, dans chaque relation, un échange se produit. Le parquet obtient l'information souhaitée, ou l'accès aux lieux ou aux publics concernés (écoles, immeubles...). L'interlocuteur, lui, voit son autorité propre renforcée en raison de son rapprochement avec le parquet. Si on prend l'exemple de l'école, le parquet informe les autorités scolaires sur le fonctionnement général de la justice, sur les suites judiciaires particulières données aux affaires concernant les élèves. Ou encore, le magistrat se rend à l'école et participe aux réunions qui y sont organisées dans le but de rappeler aux parents d'élèves un certain nombre de règles à faire respecter par leurs enfants.

À partir de là, le groupe va prendre forme et se mettre au travail. Car il ne s'agit pas seulement de travailler avec le parquet, il s'agit ensuite de travailler ensemble, pour prolonger l'action judiciaire, pour la

1. Astier, I., Revenu minimum et souci d'insertion, Paris, Desclée de Brouwer, 1997. 
compléter là où elle ne suffit pas. Et travailler ensemble, on l'a vu avec les CCPD, n'est pas évident. Trop souvent, les réunions transversales se résument à un renvoi des responsabilités des uns sur les autres. $\mathrm{Ce}$ qui se passe dans les GLTD est à cet égard nouveau, et c'est l'autre aspect de la magistrature sociale. On n'y prend pas forcément, à chaque fois, de grandes décisions; on n'y élabore pas nécessairement d'ambitieux projets concertés. Ce qui se passe, plutôt, c'est la poursuite de cette évolution des positions respectives. L'ordinaire des réunions, ce sont des échanges d'informations, la mise en commun des diagnostics, la présentation des modes d'intervention de chacun, avec ses réussites et ses contraintes. Le parquet est le premier à exposer de façon détaillée, pédagogique, son fonctionnement, à rendre compte de ses réponses, et à montrer que sa présence est assurée. Chaque acteur fait de même. A partir de là, sur fond de l'opération de "ré-assurance" initiée par le parquet, et d'une meilleure connaissance des autres, chacun reprend en quelque sorte confiance en ses propres forces, et est peu à peu conduit à " en faire plus " dans son domaine. Léchange généralisé, ainsi, institue l'ensemble des partenaires en une autorité collective, capable de prendre des décisions, d'engager des actions, mais surtout de former un réseau de gens qui se tiennent mutuellement face à leurs difficultés respectives, en puisant leur force auprès de l'instance qui les réunit au départ, le parquet.

Qu'il s'agisse de travailler avec le parquet, ou de produire ensemble de la sécurité, on a donc une évolution de la position des différents acteurs non pénaux. Mais il ne s'agit pas là d'une inféodation à une logique qui ne serait que répressive. Cette évolution n'est permise que par le fait que le parquet, de son côté, élargit lui aussi ses préoccupations, prenant au sérieux celles de ses partenaires, qui ne sont pas forcément limitées à la délinquance au sens judiciaire du terme. En deux mots, on dira qu'un type particulier de relation entre les différents partenaires, parquet et autres, a pour conséquence une tendance au partage de préoccupations qui jusque-là demeuraient clivées selon une ligne de démarcation à peu près étanche. Il ne s'agit plus soit de réprimer soit de prévenir. Ce qui est en jeu, c'est le traitement du territoire : de la poursuite des infractions, on passe à l'intérêt pour tout ce qui, bénin ou grave, fait la qualité de l'espace et, par là, permet la vie en société.

\section{Le souci du territoire}

De quoi parle-t-on dans les réunions des GLTD? Scène commune, d'abord lieu d'échanges, le groupe est l'endroit où se croisent des images. Certes, les réunions commencent par le traditionnel relevé des chiffres de la police et du parquet : délinquance de voie publique, avec son lot de vols, vols à la roulotte, dégradations et agressions, taux d'élucidation, trafics démantelés, opérations anti-drogue... Mais très vite le regard s'affine, les catégories juridiques prennent chair. Des allers-retours s'installent, entre ces données chiffrées, l'évolution positive ou négative qu'elles sont supposées refléter et la façon dont chaque participant " sent " le quartier. A partir de là, on s'aperçoit que cette évocation de l'extraordinaire n'a au fond qu'une fonction d'ouverture. De toute évidence, dans ces quartiers, c'est l'ordinaire qui fait problème. L'ordinaire, c'est-à-dire les espaces qui perdent leur qualité par la multiplication des incivilités : les halls d'immeubles encombrés de jeunes "ventouses" plus ou moins agressifs, les cages d'escalier maculées ou régulièrement privées d'éclairage, les papiers gras jonchant les abords des immeubles, les sorties d'école et l'agitation qui les entoure, telle portion de rue où se multiplient les accidents de la circulation, telle station de RER où ont élu domicile des individus plus ou moins inquiétants. L'ordinaire, partant de la répétition des délits, ce sont les conditions qui la favorisent : l'existence de lieux impossibles à surveiller, soit parce qu'ils ne sont pas éclairés ou que l'éclairage y est particulièrement vulnérable, soit en raison de coursives permettant à tout délinquant de s'enfuir sans encombre lorsqu'arrive la police, ou bien, inversement, d'impasses où l'on se sent exposé, dans l'incapacité de fuir un éventuel agresseur. L'absence de magasins, avec leur fonction de réassurance, la familiarité qu'ils donnent

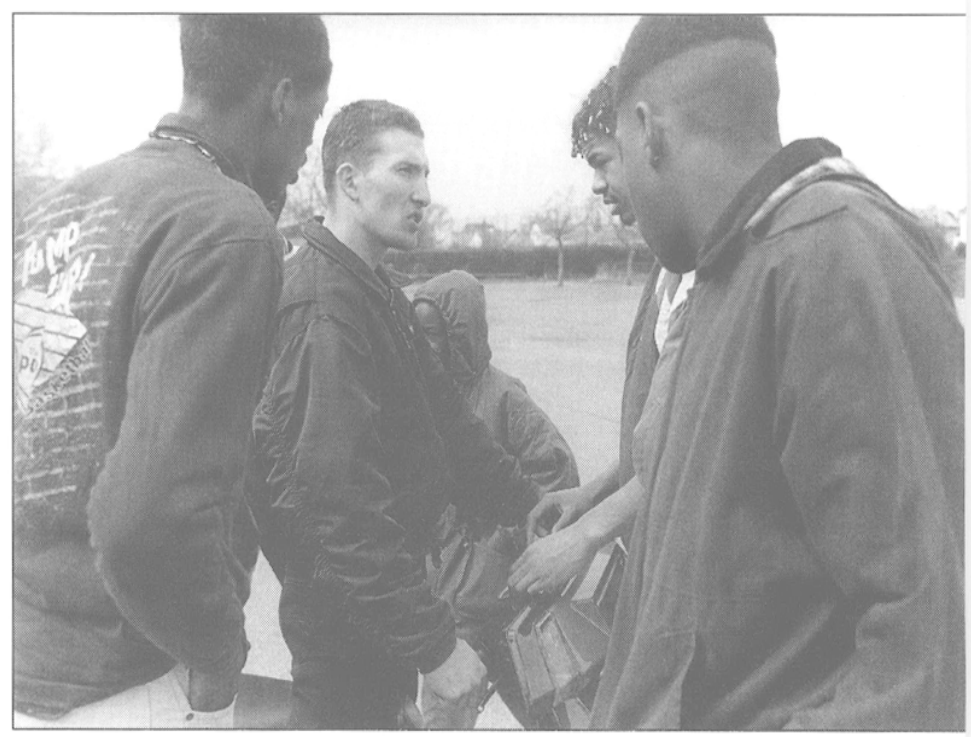

"Le Blanc-Mesnil, c'est moi. "

aux lieux, est souligné. Avec une mention spéciale pour les tags sur les murs, dans les escaliers, qui donnent le sentiment que l'espace est marqué, signalé comme le territoire des autres, de ceux qui occupent le dehors et poussent ceux qui n'en font pas partie à rentrer au plus vite chez eux. Les représentants d'associations d'habi- 
tants insistent sur cette peur qui surgit lorsqu'il faut rentrer chez soi après le dernier bus, en ayant à traverser la cité, sur la tendance qui en résulte à ne plus sortir le soir de peur de n'oser rentrer autrement qu'en taxi. La peur est le leitmotiv des discours. Elle sert à décrire la solitude du conducteur de bus, celle de l'enseignant qui quitte le collège en s'étant fait quelques ennemis parmi ses élèves, celle du collégien qui redoute d'être racketté. Il y va d'une dégradation ordinaire de la confiance, d'une perte d'aisance dans l'espace, dans les espaces intermédiaires du moins, dans la part commune du territoire.

Ces problèmes ne relèvent pas, stricto sensu, de la compétence du parquet. Celui-ci ne connaît en principe que des individus auteurs d'infractions pénales. $\mathrm{Ce}$ sont des problèmes qui relèvent d'une connaissance du territoire, d'un souci de celui-ci, qui apparait comme étant le véritable enjeu du GLTD. Tout se passe comme si la répression était un moyen, un prétexte à la limite, pour engager une action sur ce qui ne relève pas de la justice mais dont personne ne peut s'occuper sans elle. Sans doute est-ce là l'essentiel de l'impact des GLTD : cette conversion du regard que les responsables de l'action publique portent sur un quartier. Perçu au départ comme un contenant indifférencié, un simple réceptacle de délinquants, le quartier est progressivement analysé comme un espace pratique, un support concret de pratiques sociales, entaché par la gêne qui résulte de la condensation de conduites inciviles.

\section{Un espace fusionnel et privatisé}

Pour expliciter ce rôle du territoire, nous avons repris l'analyse qu'en a faite Hannah Arendt à partir de l'image de la table commune. Pour $\mathrm{H}$. Arendt, partager un espace suppose de s'établir avec les autres dans une relation où l'on est à la fois relié et séparé d'eux. Comme lorsqu'on se trouve autour d'une table : les gens se distribuent autour d'elle, et peuvent ainsi se donner à voir et à entendre les uns aux autres (reliés), tout en gardant leurs distances (séparés) puisque cet élément s'intercale entre eux. L'espace public joue en quelque sorte le rôle de la table, à l'échelle de la vie dans un quartier. Pour qu'il puisse relier les gens les uns aux autres, pour qu'une vie sociale commune soit possible, il faut d'abord qu'il les sépare, c'est-à-dire qu'il instaure entre eux le respect, qu'il les amène à se tenir à distance " respectable " les uns des autres.

Or, et c'est ce qui ressort du diagnostic fait avec des associations d'habitants, un espace qui se dégrade est un espace qui perd de sa capacité à imposer cette retenue : on y assiste à une emprise des plus forts, à des effets de dépendance, de caildat. On observe une occupation exclusive du territoire, des lieux communs, en vertu d'une logique mafieuse, où la loi du plus fort s'exprime aussi comme la loi du même.

On a évoqué la configuration de certains sites, faits de coursives et d'impasses, et dès lors impossibles à surveiller et encore plus à investir par la police. Ceux qui "tiennent " le quartier utilisent ces faiblesses, en créent d'autres : l'éclairage public est neutralisé, un portillon se trouve systématiquement détruit pour forcer un passage (ce qui conduira un habitant à déplorer que l'on persiste à préférer l'esthétique des matériaux à leur solidité). Les tags sont analysés dans cette même logique, quand un proviseur distingue les tags " signature " (les plus nombreux), que les jeunes revendiquent, avec leur dimension de marquage de territoire, des tags insultes. Dans un registre analogue, un lycée tout entier peut, malgré sa vocation régionale, être vécu par les habitants de la cité où il se trouve comme " un enjeu de territoire ", comme leur appartenant, en quelque sorte, du seul fait de sa localisation. On peut évoquer encore le dépeçage de voitures volées, devenu pratique tellement courante que ceux qui y assistent, de leur fenêtre, n'y voient plus qu'un innocent bricolage. Ou l'attitude de ce commerçant qui, agressé brutalement à plusieurs reprises par un jeune voleur à l'étalage finit par systématiquement " laisser filer les jeunes qui piquent ".

Peu à peu les habitants se trouvent plus ou moins enfermés dans une logique de connivence avec les délinquants, les trafiquants n'arrivent plus à discerner ce qui est conforme à la loi de ce qui simplement se fait. $\grave{A}$ tous les niveaux. Ici, on évoque des parents qui, dès la maternelle, ne disent plus " non " à leurs enfants. Là, les bons de voyage offerts par la mairie font l'objet d'un trafic organisé au lieu de permettre aux jeunes "bénéficiaires " de partir en voyage. Ailleurs, une rivalité entre bandes débouche sur une mort d'homme : c'est un accident. Une interpellation policière fait une victime : c'est un assassinat. Un travailleur social comparera ce quartier à une famille incestueuse, où la confusion des rôles rend difficile la constitution de repères, où les jeunes se décrivent comme victimes simultanément de la mafia et des autorités, où ils se sentent à la fois solidaires des jeunes délinquants et menacés par eux de stigmatisation.

L'autre versant de cette dégradation du territoire, c'est le repli sur le privé, chacun dans son logement, par peur d'un contact qui signifierait confusion, allégeance, dépendance. Plusieurs fois on entendra comparer l'état de dégradation des parties communes des immeubles et l'impeccable propreté, le soin apporté à l'intérieur des appartements. Le repli sur le privé consiste également, surtout, dans l'indifférence aux affaires de tous, au bien commun, le refus des gens de "se mêler de ce qui ne les regarde pas ". Les habitants répugnent à signaler les incidents du quartier, même lorsqu'ils en sont eux-mêmes victimcs. Ce repli sur le 
privé a pour conséquence une rupture de contact entre les familles et les institutions publiques. L'école est assimilée au dehors, à une partie de ce dehors vécu comme menaçant. Non que les enseignants soient d'une quelconque manière insécurisants. Mais ils appellent les parents à se comporter normalement dans une situation qui n'est pas normale. Ils veulent les sortir de leur refuge, les faire venir à l'école, au collège, pour parler de leur enfant, de ses absences, de son comportement, de son orientation. Ceux qui acceptent de se déplacer sont une minorité. Le plus souvent, ils délèguent un frère aîné, familier des lieux mais qui ne peut être considéré comme un interlocuteur valable. Le repli n'est, certes, pas total. Les locataires se connaissent, mais selon une logique très restrictive : celle de la nationalité, parfois de la religion. C'est une logique qui étend la sphère du privé, la dilate à proportion des liens qui s'établissent ainsi, mais n'établit pas un espace public.

\section{L'action du GLTD : séparer et relier}

Par rapport à cette insécurité du territoire, quelles actions sont engagées par un GLTD ? Il dispose, par principe, d'une grande concentration de moyens. Ceux de la justice, à travers le traitement en temps réel amplifié par la pratique des signalements et par la politique de systématisation; ceux de la police, dès lors qu'elle agit sur réquisition du parquet et entreprend d'intensifier son action sur l'espace en question. Et puis ceux des partenaires "sociaux", invités chacun pour son propre compte, mais aussi en s'associant, à élaborer des actions susceptibles de transformer les conditions de l'existence collective qui pèsent sur leur travail et pénalisent leurs efforts. Ce qui est nouveau, c'est que l'action du GLTD ne se réduit pas à un partage entre des actions répressives, qui seraient l'apanage des seuls acteurs judiciaires et policiers, et des actions préventives qui relèveraient des partenaires "sociaux". Ces derniers, on l'a dit, prennent leur part du travail répressif. Il en va de même, dans l'autre sens, pour la police et surtout les magistrats, qui s'associent aux initiatives des enseignants en matière de pédagogie du droit, à celles des bailleurs sociaux lorsqu'ils décident de rencontrer les locataires par cages d'escaliers ou des élus locaux pour fonder des maisons des parents.

On préfere donc, pour évoquer les actions, revenir à l'image de la table, avec sa double fonction consistant à séparer et à relier les personnes qui l'entourent. Séparer, c'est casser les logiques mafieuses qui instaurent la domination du fort sur le faible, du même sur l'autre. En ce sens, l'action de séparation ne se réduit pas à l'injonction de "circuler", d'éviter l'attroupement, les conduites suspectes, de mettre sous surveillance. Elle concerne la police, certes, mais aussi tout ce qui peut modifier l'espace en termes de sécurisation, tout ce qui peut élever sa dignité, faire qu'il apparaisse aux habitants comme une (leur) richesse commune. Pour que l'espace s'interpose entre les gens, il convient qu'il soit respectable, qu'il acquière de la valeur, que chacun éprouve son prix au lieu de le vivre comme un no man's land.

Relier, "sortir " les individus de la sphère privée où ils se réfugient, c'est tramer une reconnaissance mutuelle entre les habitants, veiller à ce qu'ils puissent faire cercle autour des institutions supposées les servir, c'est-à-dire se servir d'elles pour être ensemble. Ce n'est pas faire du lien social pour compenser celui que défait la police avec son injonction de "circuler". On dépasse ici la représentation d'une "bonne " prévention qui ferait du lien social, qui produirait de la

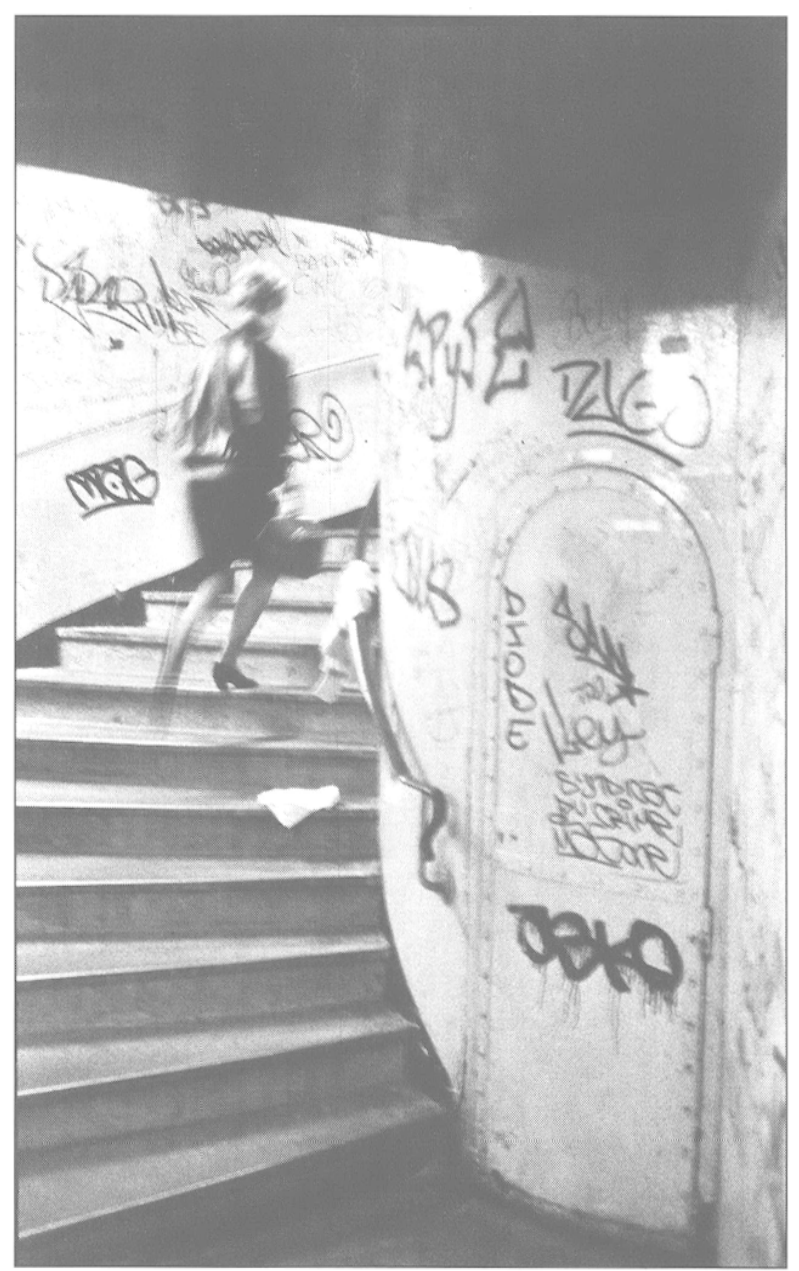

Un territoire marqué par les autres.

société, et d'une répression "mauvaise " parce qu'elle désespérerait, ne voulant voir que des individus, soit dans leur droit, soit en faute.

Pour "faire société", il ne suffit pas d'établir des liens - on n'aurait alors qu'une communauté -, il faut aussi une séparation, un vide intercalaire entre les individus. Il n'y a donc ni différence qualitative, ni hiérarchie entre les deux démarches, sinon que l'une permet 
à l'autre de fonctionner selon un principe d'affinité et non de fatalité, d'opportunité et non de hasard, de respect et non de domination.

\section{Restaurer le respect des règles et des lieux}

L'action inaugurale est purement policière, avec la multiplication des contrôles d'identité dans la soirée ou dans la nuit. À chaque fois, en effet, le bilan paraît mince, eu égard aux moyens déployés : quelques individus recherchés pour des vétilles, ou sans papiers, ou porteurs de quelques grammes de cannabis, quelques objets volés retrouvés au fond d'une cave. Il ne s'agit pas ici de déployer une logique de succès en termes de prises : l'enjeu se formule en termes de reconquête symbolique d'un territoire. Ces contrôles d'identité, ces visites de caves, opèrent un marquage du territoire public, un balisage du domaine où s'applique la loi. Le fait que la police soit systématiquement accompagnée dans ces opérations par un ou plusieurs magistrats, que ceux-ci se donnent à voir aux habitants, limite le risque de brutalités policières, de paroles vécues comme insultantes ou racistes.

Il est difficile que ne survienne pas chez les gens le sentiment de subir une agression et que cette impression n'entre en partage avec la satisfaction de voir que le quartier où ils habitent n'est plus hors-la-loi. Mais cette ambivalence elle-même est intéressante dans la mesure où elle force les habitants à réagir, où elle les amène à s'exprimer. D'autant que le magistrat se présente alors comme leur interlocuteur. Souvent " l'émotion » des habitants après plusieurs contrôles d'identité constitue l'occasion d'une rencontre entre eux et le substitut, arrangée par un élu ou un agent de développement social.

Il faut redessiner les limites de l'espace public, figurer la "table", si l'on veut que les partenaires se disposent autour d'elle.

Dans ce même esprit de renoncement à la rentabilité apparente, la police est amenée à encourager les plaintes, même pour des préjudices minimes. Cela fait baisser le taux d'élucidation des délits mais permet d'avoir une meilleure représentation de ce qui se passe, d'éviter que le territoire légal et le territoire réel ne continuent à diverger par trop. Surtout, les habitants retrouvent le sentiment que ce qui les affecte n'est pas ignoré.

Les enseignants se joignent à ce souci de restauration d'un certain nombre de repères. Ils acceptent que la police procède à des fouilles de cartables pour donner consistance à l'information préalablement prodiguée aux élèves sur l'interdiction du port d'armes. Tel proviseur appelle systématiquement la police lorsque des pitbulls vaguent à proximité de son établissement, faisant ainsi savoir à ses élèves que la loi s'applique là comme ailleurs. Ils montrent ainsi que l'école, le collège, le lycée, n'appartiennent pas au quartier, ne subissent pas la loi de ceux qui y règnent.

A cette restauration symbolique du territoire s'ajoute sa restauration matérielle. Il s'agit d'abord de colmater les brèches qui en offrent à certains la maitrise exclusive. Les bailleurs y contribuent, par des actions "lourdes" telles que le murage de caves abandonnées, lieux de recel ou de prostitution, par la fermeture des coursives permettant à un infracteur de disparaître aussitôt son agression commise, la condamnation des accès aux toits, pour décourager les guetteurs, ou encore en rendant inattaquable le circuit d'électricité, en inversant les entrées d'immeubles. Mais également au quotidien, en effaçant systématiquement les tags, façon de signifier que les halls d'immeubles, entre autres, appartiennent à tous les locataires et pas seulement aux quelques jeunes qui y passent le plus clair de leur temps. Commerçants et transporteurs peuvent être impliqués conjointement, comme dans ce projet de réaménagement d'une place pour tenter de rendre à tous l'espace attenant à une gare RER et à un centre commercial, appropriés par certains. Les établissements scolaires, eux aussi, se mobilisent contre la généralisation des graffitis, avec des opérations anti-tags qui finissent par en induire la quasi-disparition. La restauration du territoire peut également consister, là où les accidents de la circulation se multiplient, entraînant facilement des mini-émeutes, à réorganiser la voirie de manière à ralentir la circulation.

Des opérations plus complexes sont montées, dans lesquelles plusieurs acteurs additionnent leurs forces. Ainsi, dans cette cité où des espaces boueux et jonchés de détritus entourent les immeubles, la Maîtrise d'œuvre urbaine et sociale sera à l'origine d'une opération de "réappropriation du quartier" par les habitants, avec un grand nettoyage de printemps et la plantation d'arbres et de fleurs sur des parcelles mises à disposition par l'office HLM. Là également, une action au départ relativement modeste de repeinture des cages d'escaliers et d'amélioration de l'hygiène dans les immeubles, initiée par le bailleur, s'est muée en un véritable travail en partenariat sur le thème des droits et devoirs des habitants. Lors de la présentation de cette opération au groupe, on vit en effet émerger le souci d'impliquer les habitants dans la préservation des lieux une fois réhabilités, ce qui conduisit l'office à prolonger son action par la rédaction d'un document rappelant aux locataires leurs droits et devoirs, démarche relayée ensuite par les enseignants, qui proposèrent d'adapter ce texte pour $y$ travailler avec les enfants des écoles.

Symbolique, matérielle, la restauration du territoire est également morale. Des groupes de parole peuvent être institués entre jeunes et adultes sur la question des conflits qui les opposent. L'un des GLTD a été à l'ori- 
gine d'une action, en direction des 8-12 ans, consistant à réaliser " un travail sur la violence et la loi ». Des ateliers animés par la police, l'ASE, la prévention spécialisée, la PJJ, la Sauvegarde, un chargé de mission du parquet, chacun venant présenter son institution, permirent que soient abordées des questions comme ce qu'est un père, une mère, l'autorité parentale, ce que les parents ont le droit de faire ou non, le fait que la loi puisse sanctionner certains comportements aussi bien des parents que du jeune.

Plus audacieusement encore, une action a été engagée par la PJJ dans un contexte particulièrement violent puisque le site en question avait été le cadre de plusieurs meurtres, notamment celui d'un jeune du quartier, engagé dans des trafics et des rivalités de bandes. La banalisation de la mort qui s'en est suivie chez les jeunes a donné l'idée de conduire ceux-ci à s'exprimer sur la violence. L'opération était présentée comme " un parcours de création lié à un questionnement critique de la violence : celle des jeunes, celle de leur environnement ". Intitulée "Tuer n'est pas jouer", elle consistait à leur proposer de participer à des ateliers d'écriture, de création musicale, de performance scénique et préparation à l'enregistrement et comprenait une dimension de confrontation du discours des jeunes à celui des adultes « détenteurs et témoins de l'histoire du quartier".

\section{Relier institutions et habitants, parents et enfants}

Le même mouvement qui porte le GLTD à séparer ce qui s'agrège selon la loi du plus fort en profitant du retrait des individus et en produisant celui des institutions, le porte à relier ces individus et ces institutions. Il est déjà, lui-même, une réunion des institutions qui comptent sur une cité. La manifestation des moyens de la justice et de la police suffit, par l'émotion qu'elle entraine, à provoquer la réunion des habitants. Sur quels appuis le GLTD peut-il compter, pour pérenniser ses acquis au-delà de sa très courte existence, sinon ceux que constituent les réseaux, les associations qu'il aura contribué à mettre en place et qui fourniront une tramc résistante, à raison de la confiance mutuelle qui les anime, de la fonction de ressource ou/et de recours que chaque institution devient pour une autre, chaque habitant pour son voisin ? Cette activité de mise en relation se déploie sur trois niveaux : celui des institutions entre elles et avec leurs usagers, celui des habitants entre eux, celui des parents avec leurs enfants.

L'effet de mise en réseau des institutions (enseignement, logement, transport, police) avec la justice et les élus constitue le premier et le principal des bénéfices d'un GLTD. Quand un GLTD s'arrête, il est convenu de dire qu'il se trouve " mis en sommeil ». Cet euphémisme recouvre, certes, lc souci dc calmer la crainte des partenaires et surtout des élus de voir le parquet arrêter une action qui leur avait redonné confiance. Mais il constitue aussi une manière de formuler le vœu que le groupe ne s'arrête pas pour autant de fonctionner, même s'il le fait de manière moins formelle et en ne se trouvant plus sous l'égide directe du parquet. C'est un peu comme si on enlevait l'échafaudage... en espérant que la construction tiendra toute seule, que le ciment aura pris. Quand ce n'est pas le cas et, à l'évidence, cela arrive, le GLTD peut être réactivé. Ce qui fait que le réseau tient est affaire, bien sûr, de liens interpersonnels, susceptibles de se défaire par la force des mutations. Mais c'est aussi le degré d'incorporation par chaque institution de la culture du travail en réseau. Et cette culture-là est, elle-même, fonction de l'utilité que chacune des institutions a été amenée à reconnaitre à ce travail en commun pour l'accomplissement de sa propre tâche. Lorsque des enseignants ont pris l'habitude de recourir aux services du procureur pour rencontrer des parents d'élèves rétifs à tout contact, lorsque leur relation avec les îlotiers est devenue telle qu'il est " normal de voir un uniforme dans le lycée ", lorsque les bailleurs sociaux ont appris à travailler avec les enseignants pour maintenir en bon état les abords des immeubles en établissant un code des droits et des devoirs de l'office et des locataires, ou plus ponctuellement pour déplacer des containers jugés trop proches de la sortie d'une école, lorsque les transporteurs ou les postiers ont pris l'avis des habitants pour établir leurs trajets, leurs horaires, leurs localisations, ils ont intégré le principe du réseau en même temps qu'ils ont modifié leur propre mode de fonctionnement. Pourquoi, alors, le réseau ne durerait-il pas autant que de besoin?

La plupart du temps, l'apparition d'un GLTD s'accompagne de la création d'associations de locataires ou d'habitants. Dans ces cités où la loi du plus fort le dispute à celle du silence, demander aux gens qu'ils prennent en charge les affaires de la cité, qu'ils s'assument, s'unissent pour endiguer la délinquance des mineurs, veiller à la bonne marche des services, est une démarche aussi fréquente que vaine. Par contre, l'expérience des GLTD montre que partout où les institutions prennent une initiative, les habitants suivent, en profitent pour relever la tête, créer des associations, se constituer en interlocuteurs pour elles. Soit de manière réactive, soit par opportunité. On a évoqué plus haut les réunions d'habitants qui surgissaient par réaction aux contrôles de police. Hostiles au départ, les associations le sont restées jusqu'à la fin. Non par refus de la police, mais à raison d'un sentiment profond que les institutions s'entendent entre elles mais n'entendent pas les habitants. Frustration durable, auto-entretenue, mais qui n'empêche que se soient établis entre les habitants une circulation de l'information, un appui mutuel qui modifient le climat de peur et de repli sur 
soi qui régnait auparavant. Parfois, des associations surgissent en même temps que le GLTD, par un simple effet de permission implicite, de levée des inhibitions que produit celui-ci. Ces associations-là participent vite aux réunions des groupes. Elles y apportent souvent une connaissance des lieux et des problèmes qui époustoufle les institutionnels, au point parfois de les indisposer en voulant par trop guider leur action.

Enfin, s'agissant de lien, il est difficile de ne pas conclure sur celui des parents avec leurs enfants. Tout le problème de l'insécurité partirait de là, de la déliaison de ce rapport-là, de la faillite de l'autorité, de la démission des parents. Effectivement, là se situe l'horizon du GLTD, sa cible profonde et d'autant plus difficile à atteindre. Sur le plan des relations individuelles, le souci d'exiger la présence des parents là où trop souvent ils sont absents ou " représentés " par les grands frères ou grandes sœurs est manifeste. Fortement affirmé devant les délégués du procureur, à l'école, le mouvement gagne les différents acteurs institutionnels qui tous, peu à peu, intègrent cette préoccupation pour la "remobilisation" des parents. Ainsi, certains bailleurs sociaux refusent-ils d'avoir affaire aux enfants seuls pour ce qui concerne les logements. Dans une démarche plus globale, les magistrats, la PJJ, les élus, parfois les enseignants, œuvrent par ailleurs à la création de maisons des parents. Il s'agit de lieux où les parents peuvent venir parler des problèmes qu'ils rencontrent avec leurs enfants, avec l'école, avec la police et la justice. Dans des cités où de nombreuses familles sont monoparentales, l'enjeu est d'importance. La défaillance dans l'exercice de l'autorité parentale constitue une cible bien connue du parquet de Bobigny. La médiatisation de procès faits à des parents coupables de "nonchalance éducative " est aussi l'effet d'une présentation tronquée de la démarche. Témoin, cette réunion organisée, après coup, par la substitut ayant engagé les poursuites, pour expliquer aux femmes maliennes de la cité des 4000 , à La Courneuve, aussi bien l'intervention elle-même que la politique générale du parquet. Au cours de cette réunion, les femmes ont eu l'occasion d'expliquer leur vision des rapports qu'elles entretiennent avec l'école, avec la société française en général. Le tout débouchant sur la présenta- tion du projet local de maison des parents, l'action répressive, revendiquée, s'inscrivant explicitement dans un projet complexe. L'effort essentiel porte sur la reconstitution d'une parentalité trop éclatée, trop incertaine de son autorité, des moyens et des buts de celleci. Restituer aux parents le sentiment de la légitimité de leur fonction, leur transférer une part de celle du magistrat paraît plus névralgique que sanctionner leur défaillance.

\section{Garantir un territoire qui permette le respect de soi}

Lorsqu'ils entrent sur la "scène commune" du GLTD, les acteurs sont encore comme distribués en deux camps : les prestataires de la sécurité et ses usagers. Faire évoluer la position des partenaires " sociaux " revient à leur faire partager, au moins partiellement, le point de vue des prestataires de sécurité, qui est celui de l'ordre public. Il ne s'agit pas pour autant ni d'une inféodation des premiers aux seconds, ni d'un jeu de défausse de l'État sur ses partenaires. En effet, l'engagement de ceux-ci n'apparaît se réaliser que parce que leur démarche a sa réciproque : le parquet, maître d'œuvre du dispositif, montre qu'il est prêt, de son côté, à élargir le spectre de son intervention à ce qui préoccupe d'abord ses partenaires, et qui n'est pas naturellement de son ressort à lui : le quotidien, l'ordinaire, les incivilités. C'est sur le "territoire" que s'ancre cette préoccupation, c'est à partir de lui qu'on peut l'analyser. Dans une perspective qui dépasse de loin, on l'a compris, ce que l'on appelle communément prévention situationnelle. La défense de l'espace, sa protection s'élargit à sa restauration, qui elle-même n'est autre que celle des conditions de possibilité d'une vie sociale. Initié par la mobilisation des acteurs institutionnels, la démarche a pour visée ultime, à travers la relégitimation de ceux-ci, de permettre aux habitants de réinvestir le territoire, c'est-à-dire de retrouver, sous la forme d'une reconnaissance de leurs droits et devoirs, le respect aussi bien d'eux-mêmes que des autres.

Anne Wyvekens

Anne Wyvekens est chargée de recherche au CEPEL (Centre d'études des politiques sociales)/CNRS (Montpellier). Cet article reprend une recherche réalisée avec Jacques Donzelot, pour le compte de la Mission de recherche Droit et Justice, de l'Institut des Hautes études de la sécurité intérieure et de la Délégation interministérielle à la ville : La politique judiciaire de la ville : de la prévention au traitement. Les groupes locaux de traitement de la délinquance, Centre d'études des politiques sociales, décembre 1998. Cette recherche a été publiée également dans I. Donzelot, A. Wyvekens, «Magistrature sociale et souci du territoire. Les groupes locaux de traitement de la délinquance ", in Les partages de la sécurité. Les Cahiers de la Sécurité intérieure, n³3, 1998, pp. $149-174$. 